\title{
Research into purchasing health care: time to face the challenge
}

\author{
Gilles de Wildt, Raj Bhopal, Mike Lavender
}

Purchasing health care by district health authorities (or health commissions) and fundholding general practices in the UK is of great importance for health and health care delivery and deserves deeper study. In the face of the difficulties and challenges in undertaking research on this subject, we wish to make some suggestions on how research should proceed, and to appeal for a research programme commensurate with the importance of the topic.

To study a subject effectively requires clarity of understanding and definition. However, the concept of health gain through purchasing health care in a managed market is complex, changing, and fluid. ${ }^{1}$ Purchasing is at the core of the reforms of the NHS in the UK but the reforms themselves lack clarity in terms of direction and purpose, being effectively "made up as they go along". ${ }^{2}$ In the absence of appropriate precedents, ${ }^{13}$ experimentation with different models of purchasing will be needed. General practice fundholding, which may soon cover one fifth of government health care expenditure, is creating new relations between general practitioners and hospitals and other providers ${ }^{34}$ but its overall impact remains unclear. Purchasing of health care by health authorities (or health commissions) is still at an early stage of evolution, and actual changes in the services commissioned and delivered have been small. ${ }^{15}$ Researchers therefore have to operate in the absence of a solid past foundation of knowledge about health care purchasing ${ }^{2}$ and uncertainty about the future direction and pace of change.

The speed of the reforms is so great that research is hard to carry out. By the time any report is published, the situation reported on may not exist. Furthermore, the effects of health care purchasing demonstrated at one time and place may not apply in the future or in different places. Either the research needs to be speedier and reporting mechanisms smoother, or the reforms need to be signalled well in advance or slowed down.

The lack of clarity about the meaning and goals of purchasing in health care poses a major problem for research. Researchers need to carefully describe and analyse the different meanings of "purchasing" at different levels in the health service, and the actual practice of commissioning agencies and fundholders. They need to make explicit what are at present implicit expectations and aims. For example, at a policy level purchasing has two key elements that have clear operational objectives. The first element is the separation of purchasing from the provision of health care services. This involves the commissioning of health services for a population on the basis of measured health needs within the limits of allocated resources. The second element is the allocation of health service resources using alternative models for purchasing - the district health authority (or health commission) and GP fundholders in their various forms. However, in practice there are many interpretations of the meaning of purchasing and the intended outcome remains unclear. Is it to promote competition or increase efficiency? Is it to meet the need for health care or control the demand for health services? Is it to promote better health or to improve the quality of health care? As it is probable that the goals of purchasing include all of these, research needs to be broad in its focus and yet specific in the statement of research questions.

Purchasing forms an inseparable part of the health reforms in the UK, which, in turn, cannot easily be disentangled from other profound changes in UK society. These changes include widening socioeconomic differences and changing labour relations between the state and health professionals. ${ }^{6}$ Arguably, these changes may have greater influence on health and health care than NHS reforms. It will be difficult to develop plausible models which can incorporate and adjust for such influences.

The reforms have created changes within the NHS that have produced problems of access to data collected by providers about patient care, about policies, and about organisational changes. Providers may perceive this information as commercially sensitive, rather than as information which should be shared and discussed among professionals, managers, patients' representatives, government and researchers. There is evidence of the emergence of a culture of secrecy in UK health care. ${ }^{78}$ Other developments may compound this problem further, such as the recent change to civil servant status of regional directors of public health and increased concerns about job security by professionals, including public health professionals, in a service which goes through repeated reorganisations and which may develop a tendency to self censorship. ${ }^{9}$ Successful research requires openness.

In the face of these hurdles, what can be the role of research? Currently, policies are not sufficiently informed by research. ${ }^{10}$ The UK government has not encouraged or commissioned a large scale evaluation of the NHS reforms, (although researchers have tackled component parts ${ }^{11-13}$ ) and is unlikely to call a halt to further organisational change to allow a proper evaluation to take place. ${ }^{7}$ The expansion of GP fundholding and the development of total purchasing pilot sites is a clear indication of the government's preferred model for purchasing. ${ }^{413}$ However, the lack of 
comparative research into the effects of different models of purchasing means that the policy lacks research evidence on the costs and benefits of this approach. The key question for which there is no evidence is whether making purchasing decisions "as close to the patient as possible" is more responsive to meeting the health needs of a population than commissioning services by a district health authority? There has been very little research on the impact of fundholding that include nonfundholding practices as controls. ${ }^{14}$ The evaluation of total purchasing pilot sites will be a comparison with standard fundholding and misses the opportunity to assess the alternative approaches to purchasing. ${ }^{15} \mathrm{With}$ the expansion of fundholding a priority for health authorities in the medium term, ${ }^{16}$ it will be difficult to design an experiment that includes non-fundholding practices as controls during a period of change. ${ }^{17}$ The alternative design using practices as their own control over time is feasible but less convincing for, as stated earlier, there are many other profound changes occurring in medical knowledge and social organisation which confound associations between changes in health care and fundholding.

Whatever the direction and speed of change of the NHS reforms, the potential effects of purchasing are too important to cast it aside as a research topic. Therefore, research into this field needs to be declared a NHS research priority, and funds and manpower need to be identified, followed by a call for proposals. At the national level a multidisciplinary research effort is required, not only to develop conceptual frameworks and appropriate, explanatory models, but also to carry out initial observational quantitative and qualitative studies of different elements of purchasing. Importantly, there should also be research into the policies which drive the reforms. ${ }^{10}$

To help achieve research-based policy formulation, the Dutch example, with its scenario studies, might be followed. ${ }^{18}$ These have been applied to possible changes in health service organisation, to health care for population groups such as the elderly, ${ }^{19}$ and for specific conditions. ${ }^{20}$ Scenario studies aim at exploring possible future developments in health and health care under different sets of assumptions on the financing and organisation of health care, medico-technological breakthroughs, and epidemiological patterns of disease. Scenario studies involve a multidisciplinary approach, the extraction of expert opinion, and modelling. This method may be suitable to assess the likely impact of purchasing policies.

Small scale research, both quantitative and qualitative, should proceed at the local level to describe, analyse, and guide health care purchasing. Valid, practical indicators are needed which can give insight into issues of equity and other aspects of quality of care for use in districts and localities. Local research may be aimed at improving the processes by which purchasing for health gain occurs. Associations between changes in purchasing policies or practices and other aspects of health and health care delivery need to be interpreted cautiously until there are clear conceptual frameworks and plausible models which incorporate other influential aspects of our fast and intensively changing society. Attributing changes as causally related to purchasing or other elements of the reforms will be particularly difficult in small scale, local research.

The obstacles to research in health care purchasing are formidable, but its importance demands the independent, focused and concerted efforts of the most talented health services researchers, encouraged by policy makers at the highest level.

\section{Summary}

Purchasing health care is at the core of the reforms of the UK NHS and yet there is little research evidence on which the policy is based. Research in this area is hampered by a lack of clarity over the aims of purchasing and the pace of change within the NHS. Purchasing developments such as general practice fundholding are proceeding without a large scale evaluation of their impact. At a national level a research effort is required to investigate this key area of the NHS reforms.

1 Øvretveit J. Purchasing for health. A multidisciplinary in troduction to the theory and practice of health purchasing. troduction to the theory and practice of health

2 Ham C. Where now for the NHS reforms? Making them up as they go along. BMF 1994;309:351-2.

3 Light DW. Managed care: false and real solutions. Lance 1994;344:1197-9.

4 Dixon J, Glennerster $\mathrm{H}$. What do we know about fundholding in general practice? BMF 1995;311:727-30.

5 Donaldson L. Building Quality into contracting and purchasing. Quality in Health Care 1994;3:S37-40.

6 Harrison S, Pollitt C. Controlling health professionals. The future of the work and organisations of the NHS. Buckingham: Open University Press, 1994.

7 Anonymous. The real lessons from Plymouth, Burnley, Brighton. Lancet 1994;344:831-2.

8 Bradlow J, Coulter A. Effect of fundholding and indicative prescribing schemes on general practitioners' prescribing prescribing schemes on general

9 Craft N. Sheard S, Smith R. The rise of Stalinism in the NHS. $B M \mp$ 1994;309:1640-5.

10 Ham C, Hunter DJ, Robinson R. Evidence based policy making. $B M \mathcal{F}$ 1995;310:71-2.

11 Robinson R, Le Grand J eds. Evaluating the NHS reforms. London: Kings Fund, 1994

12 Bradlow J, Coulter A. Effect of fundholding and indicative prescribing schemes on general practitioners' prescribing costs. BMF 1993;307:1186-9.

13 Howie JGR, Heaney DJ, Maxwell $M$. Evaluating care of patients reporting pain in fundholding practices. $B M \mathcal{F}$ 1994;309:705-10.

14 NHS Executive. Developing NHS purchasing and GP fundholding. Leeds: NHSE, 1994. (EL(94)79).

15 Coulter A. General practice fundholding: time for a cool appraisal. Br f Gen Pract 1995;45:119-20.

16 Ham C, Shapiro J. The future of fundholding. BMF 1995; 310:1150-51.

17 NHS Executive. Priorities and planning guidance for NHS 1995/96. Leeds: NHSE, 1995. (EL(95)68).

18 Howie JGR. Heaney DJ. Maxwell M. Care of patients with selected health problems in fundholding practices in Scotland in 1990 and 1992: needs. process and outcome. Br F Gen Pract 1995;45:121-26.

19 STG. Scenarios and other methods to support long term health planning. Utrecht, Netherlands: Jan van Arkel Publishers, 1987.

20 STG. Growing old in the future. Dordrecht, Netherlands: Kluwer Academic Publishers, 1987.

21 STG. Future scenarios on dental health care. A reconnaissance of the period 1990-2020. Dordrecht, Netherlands: Kluwer Academic Publishers, 1994. 